\title{
Ictiofauna de descarte en la pesca artesanal de camarones (Litopenaeus vannamei y L. stylirostris) en la laguna costera Mar Muerto, Golfo de Tehuantepec, México
}

\section{Fish discard in artisanal fishery of shrimp (Litopenaeus vannamei and $L$. stylirostris) in the Mar Muerto coastal lagoon, Gulf of Tehuantepec, Mexico}

\author{
Jonny A. Hernández-Roque ${ }^{1}$, Emilio I. Romero-Berny ${ }^{1}$ * yesús M. López-Vila
}

\begin{abstract}
RESUMEN
Se realizó una caracterización de la ictiofauna de descarte asociada a una pesquería artesanal no regulada de camarón en la laguna costera Mar Muerto, Golfo de Tehuantepec, México. Se realizaron muestreos de las capturas de camarón de octubre 2017 a enero 2018 con redes de copo fijas. En este estudio el descarte integró un porcentaje promedio de $64.2 \pm 15.3 \%$ de la biomasa total capturada. De este descarte, el grupo de los peces integró entre el 51 y $79.5 \%$, estimándose una proporción general de ictiofauna/camarón de 1.89:1 kg. No se encontró una relación entre la captura de camarón y la biomasa de ictiofauna de descarte. Altos valores de diversidad se presentaron en los meses de noviembre y diciembre, debido a una mayor presencia de individuos de afinidad marina. Se demostró que la pesca por copo afecta potencialmente por lo menos a 73 especies de peces, que de acuerdo a su talla media y especie $(9.9 \pm 9 \mathrm{~cm}$, longitud patrón), pueden corresponder a individuos juveniles y subadultos. Las familias mejor representadas en el elenco íctico fueron Carangidae, Ophichthidae y Gerreidae. Aunque se reporta una riqueza relativamente alta en el descarte, solo 4 especies fueron dominantes. Se destaca la adición de 15 nuevos registros de peces a la ictiofauna conocida en el contexto local y regional. Es necesario realizar caracterizaciones adicionales sobre fauna de acompañamiento y descarte para proponer acciones de manejo y reducción del impacto pesquero.
\end{abstract}

Palabras claves: Comunidad de peces, fauna de acompañamiento, biomasa, riqueza específica, diversidad

\section{ABSTRACT \\ Discarded ichthyofauna associated to unregulated artisanal shrimp fishery in the Mar Muerto coastal lagoon, Gulf of Tehuantepec, Mexico, was characterized. Shrimp catches were sampled \\ 1 Centro de Investigaciones Costeras, Instituto de Ciencias Biológicas. Universidad de Ciencias y Artes de Chiapas. J. J. Calzada y Calzada de Guadalupe, Col. Evolución. CP. 30700. Tonalá, Chiapas, México; jonny.hero10@gmail. com, emilio.romero.ex@unicach.mx*,chus_3f@yahoo.com.mx}


from October 2017 to January 2018 with fixed cod-end nets. In this study, discard averaged 64.2 $\pm 15.3 \%$ of the total biomass captured, while fish amounted to $51 \%$ to $79.5 \%$ of this discard, with an estimated general ichthyofauna/shrimp ratio of 1.89: $1 \mathrm{~kg}$. No relationship was found between shrimp catch and discard ichthyofauna biomass. High values of diversity were recorded in November and December due to a greater presence of marine organisms. It was shown that cod-end net fishing potentially affects at least 73 fish species, which, according to their average size and species $(9.9 \pm 9 \mathrm{~cm}$, standard length), may be juvenile and sub-adult individuals. The families best represented in the fish list were Carangidae, Ophichthidae, and Gerreidae. Although a relatively high richness is reported in the discard, only 4 species were dominant. Fifteen new records of fish were added to the known ichthyofauna in the local and regional context. Further characterizations on by-catch and discards are necessary to propose management actions and reduction of the fishing impact.

Keywords: Fish community, by-catch, biomass, specific richness, diversity

\section{INTRODUCCIÓN}

La captura incidental o secundaria de especies en las artes de pesca representa en la actualidad uno de los principales problemas que impacta a los ecosistemas acuáticos (Soykan et al. 2008). Esta captura incluye a organismos que son distintos a los del objeto de la pesca $\mathrm{y}$, que por motivos económicos, legales o personales (Alverson et al. 1994), son lanzados fuera de borda (ya sea vivos o muertos) para ser regresados al ambiente acuático; a esto se le conoce como descarte (FAO, 1996; Eayrs, 2007; Zeller et al. 2017). Se ha demostrado que la mayor parte del descarte se integra por peces, que en el caso de las lagunas costeras pertenecen a estadios juveniles, al ser estos sistemas sitios de reproducción y crianza para muchas especies (Day et al. 1989). Por lo tanto, la mortalidad de organismos capturados incidentalmente afectará a aquellos que no han alcanzado la talla de primera madurez sexual. Así mismo, muchas especies raras que ingresan a las lagunas costeras para alimentarse y que presentan bajas tasas reproductivas (p. ej. Condríctios), también suelen perecer en el descarte (Hall et al. 2000; Tsagarakis et al. 2017).

Los camarones peneidos constituyen uno de los recursos pesqueros más importantes en las zonas costeras tropicales y subtropicales (Pérez-Castañeda \& Defeo, 2001). Una característica de algunos peneidos es su ciclo de vida anfibiótico, que incluye una fase juvenil-subadulta asociada a los estuarios y explotada por la pesca artesanal, así como una fase adulta ligada al ambiente marino, explotada por la pesca industrial (García \& Le Reste, 1986). Se ha reportado que la pesquería industrial o semiindustrial de camarones, principalmente la de arrastre, genera las mayores cantidades de fauna acompañante en el nivel mundial 
(Gillett, 2008). Por lo tanto, diversas investigaciones se han llevado a cabo sobre la composición y volumen del acompañamiento y descarte, lo que permite incrementar el conocimiento taxonómico de la fauna demersal (e.g. Herrera-Valdivia et al. 2016; Giménez-Hurtado et al. 2016; Morán-Silva et al. 2017).

Para las costas de México se cuenta con datos sobre fauna acompañante y descarte generada por arrastres en las principales regiones de producción pesquera de camarón, como lo son el Golfo de Tehuantepec (Tapia-García y García-Abad, 1998), el Golfo de California (López-Martínez et al. 2010; López-Martínez y Morales-Bojórquez, 2012; Herrera-Valdivia et al. 2016) y el Golfo de México (Wakida-Kusunoki et al. 2013; Morán-Silva et al. 2017). Sin embargo, para el caso de la pesca artesanal ribereña este aspecto ha sido escasamente estudiado (Amezcua et al. 2009; Burgos-León et al. 2009; Poot-Salazar et al. 2009), a pesar de que se ha estimado que más del $30 \%$ de la pesca total de camarón en México proviene de los ambientes costeros continentales (Contreras, 2002).

El arte de pesca para camarón utilizado y regulado por la normatividad mexicana en lagunas costeras (NOM-022-PESC) es la atarraya, la cual debe observar una luz de malla de $38.1 \mathrm{~mm}$. Además de la atarraya se utilizan también los llamados "copos camaroneros", cuyo uso representa una de las formas de pesca ilegal más comunes en los sistemas lagunares del Golfo de Tehuantepec (Medina-Reyna, 1999). Básicamente, el copo camaronero es una modificación de una red de fondo con forma cónica o cilíndrica, pero en lugar de requerir un arrastre mecánico, es fijado mediante estacas con la boca a contracorriente. La captura total se concentra al final de la red y es retenida mediante trampas a manera de embudos a lo largo de la misma (Ramos-Cruz, 2011). Este arte de pesca suele utilizarse en canales de marea por donde se desplaza el camarón durante ciertos periodos. Debido a su reducida luz de malla, y a que aprovecha la velocidad de corriente, el copo es un arte de pesca de baja selectividad que puede representar proporciones de descarte de hasta $5: 1 \mathrm{~kg}$ con respecto al camarón capturado (López-Vila, 2010).

Uno de los principales componentes del descarte, tanto en pesquerías de escama como de camarón, recae en el grupo de los peces (Bojórquez, 1999; Rodríguez-Romero et al. 2009; Uzer et al. 2017). Desde un punto de vista funcional este grupo de vertebrados se considera crítico al ocupar diversos niveles tróficos y ser potenciales indicadores de la integridad ecológica de los sistemas acuáticos (Huidobro, 2000; Ramírez-Herrejón et al. 2012; González-Zuarth \& Álvarez, 2014). Aunque cierto porcentaje de peces capturados incidentalmente con el 
camarón son aprovechados, un considerable volumen es desechado (en su mayoría muertos) como descarte al carecer de valor comercial. Esto puede significar un impacto en la dinámica poblacional de muchas especies, alterando la estructura de la comunidad íctica (Hutchings, 2000, Herrera-Valdivia et al. 2016; Morán-Silva et al. 2017). Debido a la escasa información que existe sobre el descarte asociado a la pesca artesanal ribereña de camarón, en este estudio se planteó el objetivo de caracterizar la composición ictiofaunística del descarte, sus indicadores biométricos (talla y peso) y su frecuencia de aparición; así como la contribución como componente de la captura total y su relación con la biomasa de camarón.

\section{MATERIALES Y MÉTODOS}

\section{Área de estudio}

La laguna Mar Muerto se localiza al norte del Golfo de Tehuantepec (Pacífico Oriental Tropical), entre los estados de Oaxaca y Chiapas $\left(16^{\circ} 18^{\prime}-15^{\circ} 55^{\prime}\right.$ $\mathrm{N}$ y $94^{\circ} 28^{\prime}-93^{\circ} 48^{\prime}$ O; Fig. 1). Tiene una longitud de $12 \mathrm{~km}$ en su parte más ancha y unos $60 \mathrm{~km}$ de largo, por lo que es una de las lagunas costeras más extensas del Pacífico mexicano. Presenta comunicación permanente con el océano a través de la Boca de Tonalá, con una anchura aproximada de 800 m. La profundidad del cuerpo lagunar varía entre 0.5 a $6.0 \mathrm{~m}$. El régimen mareal es mixto-semidiurno con un rango promedio de $1.0 \mathrm{~m}$. En la zona de estudio prevalece el clima cálido subhúmedo tipo $\mathrm{Aw}_{\mathrm{i}}$ (w) i' $\mathrm{g}$ (clasificación de Köppen modificada; García, 2004), con menos del 5\% de la precipitación anual durante el invierno. La temperatura ambiental llega a alcanzar un máximo de $37^{\circ} \mathrm{C}$ (mayo) y una mínima de $20^{\circ} \mathrm{C}$ (noviembre) (Tapia-García et al. 2011). Se distinguen claramente dos estaciones climáticas durante el ciclo anual: lluviosa (junio-octubre) y seca (noviembre-mayo). Hacia el final de la estación lluviosa y los primeros meses de la seca, masas de aire frío provenientes del Golfo de México atraviesan el Istmo de Tehuantepec que al chocar con el aire cálido del Pacífico generan patrones de circulación anticiclónica, ocasionando fuertes vientos de descenso llamados localmente "Tehuanos", que en casos extremos pueden superar rachas de $150 \mathrm{~km} / \mathrm{h}$ (Schultz et al. 1997). Estos eventos generan corrientes superficiales y cambios en la profundidad y temperatura del agua, lo cual puede influir en la estructura de las comunidades bióticas (Tapia-García et al. 2007). Durante los meses de "Tehuanos", la salinidad del agua fluctúa de 30 a 50 PSU, mientras que la temperatura varía entre 21.5 y $38.5^{\circ} \mathrm{C}$ (Tapia-García et al. 2011). 


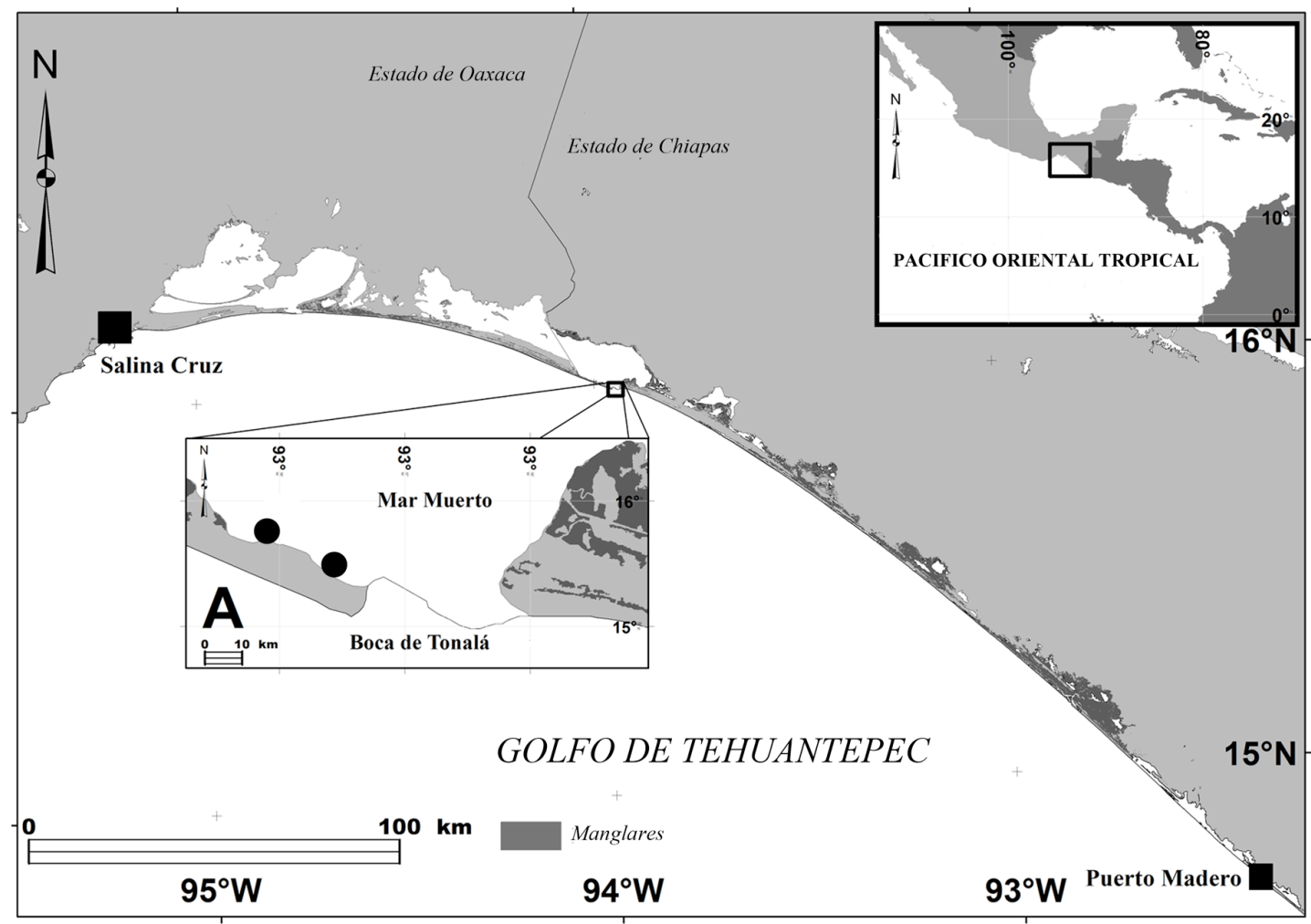

Fig. 1. Localización del área de pesca con copo (@) en la laguna costera Mar Muerto, Golfo de Tehuantepec, México

Fig. 1. Location of cod-end net fishing area (O) in the Mar Muerto coastal lagoon, Gulf of Tehuantepec, Mexico

Obtención de muestras e identificación taxonómica

Las muestras de peces se obtuvieron del descarte generado en la pesca artesanal de camarones (principalmente Litopenaeus vannamei y $L$. stylirostris) con cuatro copos camaroneros (6 x $1.7 \mathrm{~m}$ de boca, $10 \mathrm{~m}$ de longitud total y $19 \mathrm{~mm}$ de luz de malla). El área de pesca correspondió a la zona conocida como La Barra, aproximadamente a $500 \mathrm{~m}$ de la Boca de Tonalá (Fig. 1). Entre octubre de 2017 y enero de 2018 se realizaron un total de 16 despliegues (cuatro por mes), con un tiempo efectivo de pesca de siete horas por cada uno (entre las 18:00 y 1:00 hrs). La frecuencia de despliegues y el tiempo efectivo se apegaron a la práctica local de pesca con copo en la región, la cual únicamente se realiza 
durante los días de viento "Tehuano" $(>40 \mathrm{~km} / \mathrm{h})$. Se registró el peso de la captura total y posteriormente los pesos separados de camarón, descarte e ictiofauna acompañante. Del descarte de esta última muestra se obtuvieron sub-muestras homogenizadas correspondientes al $10 \%$ del peso total, que fueron congeladas y trasladadas a laboratorio. Cada organismo fue contabilizado, pesado con una balanza digital Ohaus ( $0.1 \mathrm{~g}$ de precisión) y medido (longitud patrón en $\mathrm{cm}$ ) con un ictiómetro convencional. La identificación en el nivel de especie se llevó a cabo con claves y guías para la región del Pacífico Oriental Tropical (Fischer et al. 1995; Allen \& Robertson, 1998; Castro-Aguirre et al. 1999; Miller et al. 2009; Bussing \& López, 2011). Los organismos identificados fueron fijados en formalina al $10 \%$ y lavados con agua corriente al transcurrir 48 horas. Finalmente fueron preservados en una solución de alcohol al 70\%. Aquellos ejemplares que representaron nuevos registros para la localidad de Mar Muerto (Tapia-García et al. 1998; Romero-Berny et al. 2018), para la ictiofauna continental (Velázquez-Velázquez et al. 2016) o para la ictiofauna marino-costera de Chiapas (González-Acosta et al. 2017), fueron catalogados y depositados en la colección ictiológica del Museo de Zoología de la Universidad de Ciencias y Artes de Chiapas (Registro: CHISPEC-210-03-09). La lista sistemática se arregló por orden y familia de acuerdo a Nelson (2006) y la nomenclatura fue actualizada con base en Eschmeyer et al. (2017). Se incluyó la categoría de afinidad ecogeográfica para cada especie de acuerdo a su tolerancia a la salinidad (Myers, 1949; Miller, 1966; Castro-Aguirre et al. 1999): dulceacuícola secundaria, estuarina residente, marina eurihalina y marina estenohalina.

\section{Análisis de datos}

Cada especie se categorizó de acuerdo a su Índice de Ocurrencia (Dos Santos et al. 2002) con la siguiente fórmula:

$$
S_{\mathrm{OC}}=(n i / N) 100 \text {, }
$$

en donde $S_{\mathrm{OC}}$ es el Índice de Ocurrencia, $n i$ es el número de despliegues en los cuales la especie estuvo presente y $N$ es el número total de despliegues. Las categorías se asignaron en función a los siguientes valores porcentuales:

Especies raras: Se encontraron en menos del 10\% de los despliegues.

Especies ocasionales: Se encontraron entre un $10 \%$ y $25 \%$ de los despliegues. Especies comunes: Se encontraron entre un $25 \%$ y $50 \%$ de los despliegues. Especies dominantes: Se encontraron en más del 50\% de los despliegues.

La diversidad presentada por mes se analizó con base en el índice de Shannon-Wiener $\left(\mathrm{H}^{\prime}\right)$ y el inverso 
del índice de Simpson $\left(K=\mathrm{D}^{-1}\right)($ Krebs, 2009), al seguir rutinas en el paquete PAST 3.16 (Hammer et al. 2001).

La proporción de ictiofauna de descarte y de camarón a captura total fue calculada dividiendo la biomasa de peces entre la biomasa obtenida por hora (Hall, 1996). Se utilizaron pruebas de Chi cuadrado de Pearson para contrastar las diferencias de las proporciones entre meses. Para determinar una posible relación entre las proporciones, se realizó un análisis de regresión por cuantiles $(0.75)$ con la biomasa de la ictiofauna como dependiente de la biomasa de camarón obtenidas por hora (Öndes et al. 2017), posterior a una transformación logarítmica de los datos. Los análisis estadísticos se realizaron mediante el paquete STATA/SE 11 (StataCorp., 2009).

\section{RESULTADOS}

Los datos sobre la biomasa de las capturas totales, captura de camarón e ictiofauna de descarte se presentan en el Cuadro 1. La biomasa total de camarón capturado durante el periodo de estudio fue de $620.5 \mathrm{~kg}$, con una captura media mensual de $155.12 \pm 160.61 \mathrm{~kg}, \mathrm{y}$ captura media por hora de $22.16 \pm 22.94$ $\mathrm{kg}$. La captura media mensual de ictiofauna de descarte estimada fue de $136.97 \pm 52.64 \mathrm{~kg}$, con una captura media por hora de $19.57 \pm 7.52 \mathrm{~kg}$. Los porcentajes de contribución de camarón e ictiofauna de descarte a la captura total fueron de $41.48 \%$ y $36.63 \%$, respectivamente; el $21.89 \%$ correspondió a otros invertebrados. Con respecto al patrón temporal, se observó un máximo de contribución de ictiofauna de descarte durante octubre $(68.51 \%)$, y un mínimo durante diciembre (28.88\%) (Fig. 2). En este orden, se detectaron diferencias significativas en la proporción de ictiofauna de descarte a captura de camarón de octubre con respecto a los demás meses (octubre-noviembre: $\chi^{2}=21.58, \quad P<0.0001$; octubre-diciembre: $\chi^{2}=34.01, P<0.0001$; octubre-enero: $\left.\chi^{2}=15.63, P<0.0001\right)$. La propor-

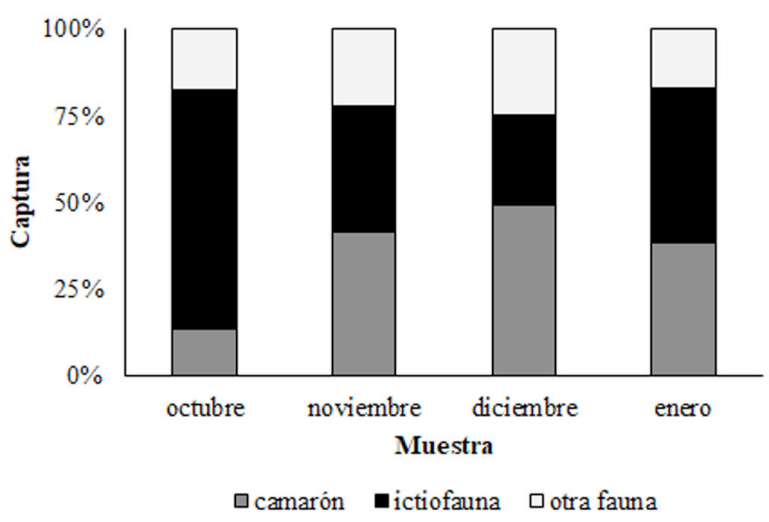

Fig. 2. Porcentaje de camarón, peces y otra fauna capturadas con copo durante el periodo octubre 2017-enero 2018 en la laguna costera Mar Muerto, México

Fig. 2. Percentage of shrimp, fish, and other fauna captured with cod-end net during the October 2017-January 2018 period in the Mar Muerto coastal lagoon, Mexico 
ción general de ictiofauna de descarte camarón y la de ictiofauna de descarte a captura de camarón fue de 0.89 , con obtenidas por hora $\left(75^{\circ}\right.$ Cuantil, Coeuna proporción promedio de 1.89:1 ficiente de regresión $=0.036, P=0.732$, (Cuadro 1). No se detectó una rela- $t=-0.35$; Fig. 3 ).

ción significativa entre la biomasa de

Cuadro 1. Indicadores de capturas totales y relativas de camarón y peces de descarte de una pesquería con copo en la laguna costera Mar Muerto durante el periodo octubre 2017-enero 2018

Table 1. Indicators of total and relative shrimp catches and discarded fish from cod-end net fishery in the Mar Muerto coastal lagoon during the October 2017-January 2018 period

\begin{tabular}{ccccccccc}
\hline Mes & $\begin{array}{c}\text { Captura } \\
\text { total }\end{array}$ & \multicolumn{2}{c}{ Captura de camarón } & \multicolumn{2}{c}{$\begin{array}{c}\text { Ictiofauna de } \\
\text { descarte }\end{array}$} & $\begin{array}{c}\text { Proporción } \\
\text { ictiofauna/ } \\
\text { camarón }\end{array}$ \\
\hline & $\mathrm{kg}$ & $\mathrm{kg}$ & $\%$ & $\mathrm{~kg} \mathrm{~h}^{-1}$ & $\mathrm{~kg}$ & $\%$ & $\mathrm{~kg} \mathrm{~h}-1$ & \\
Octubre & 191.5 & 26.5 & 13.8 & 3.79 & 131.19 & 68.6 & 18.74 & 4.95 \\
Noviembre & 205.5 & 85 & 41.4 & 12.14 & 75.55 & 36.8 & 10.79 & 0.89 \\
Diciembre & 788.6 & 389 & 49.3 & 55.57 & 204.1 & 28.8 & 29.16 & 0.52 \\
Enero & 310.2 & 120 & 38.7 & 17.14 & 137.04 & 44.2 & 19.58 & 1.14 \\
\hline
\end{tabular}

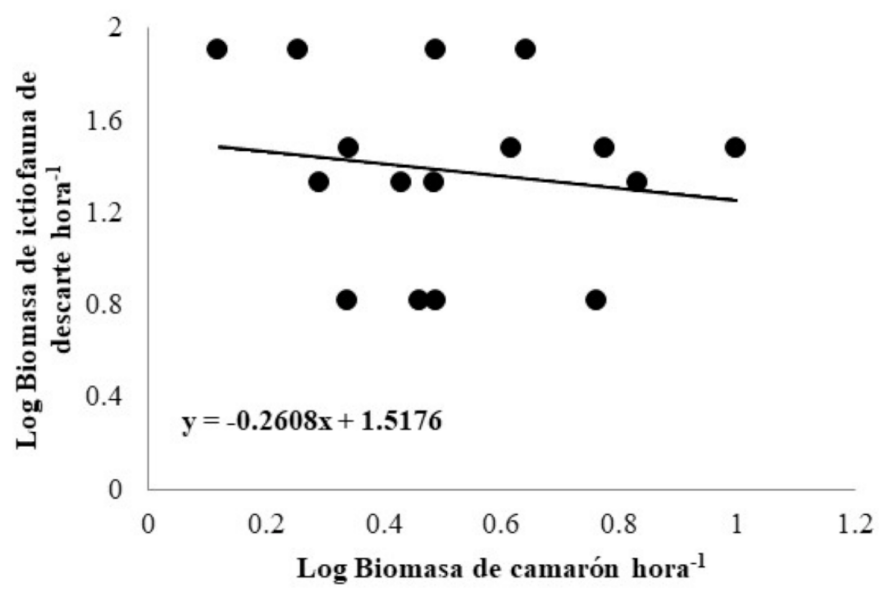

Fig. 3. Relación entre Log biomasas de ictiofauna de descarte/hora y camarón/ hora

Fig. 3. Relationship between biomass log of fish discard/hour and shrimp/hour 
Para analizar la riqueza y composición se recolectaron un total de 909 organismos con un peso total de $18.79 \mathrm{~kg}$. La ictiofauna de descarte se integró por 2 clases, 17 órdenes, 38 familias, 61 géneros y 73 especies (4 condríctios y 69 osteíctios; Cuadro 2). La familia mejor representada en el elenco íctico fue Carangidae con 7 especies, seguida por las familias Ophichthidae y Gerreidae (6 especies cada una). De acuerdo a la afinidad ecogeográfica de las especies, se encontró que el 46.6\% (34 especies) pertenecen al grupo de marinas estenohalinas, seguidas en porcentaje $(39.7 \%, 29$ especies) por las marinas eurihalinas. En menor proporción se encontraron representados los componentes estua- rino residente $(8.2 \%)$ y dulceacuícola secundario (5.5\%).

Respecto a las características biométricas estimadas, el organismo de menor peso registrado fue de la especie Achirus mazatlanus (0.479 g, con una talla de $3.6 \mathrm{~cm}$ ), mientras que el mayor peso correspondió a un ejemplar de Diodon hystrix (503 g, con una talla de $24 \mathrm{~cm})$. El organismo de menor talla fue un Prionotus horrens $(3 \mathrm{~cm}$, con un peso de $1 \mathrm{~g}$ ), correspondiendo el de mayor talla a Ethadophis byrnei (76.5 cm, con un peso de $49.7 \mathrm{~g}$ ). Con respecto al $S_{\text {oc }}$, el $45.83 \%$ fueron especies con categoría de raras, seguidas de especies ocasionales $(38.8 \%)$, y por último las comunes $(9.7 \%) \mathrm{y}$ dominantes (5.5\%) (Cuadro 2). Los

Cuadro 2. Composición de la ictiofauna de descarte de una pesquería con copo en la laguna costera Mar Muerto durante el periodo octubre 2017-enero 2018. Afinidad ecogeográfica en corchetes después del nombre de la especie (DS: dulceacuícola secundaria, ER: estuarina residente, EU: marina eurihalina, ES: marina estenohalina). N: número de organismos; LP: longitud patrón; $S_{\mathrm{OC}}$ : Índice de Ocurrencia (R: rara, O: ocasional, C: común, D: dominante)

Table 2. Composition of the discarded ichthyofauna from cod-end net fishery in the Mar Muerto coastal lagoon during the October 2017-January 2018 period. Ecogeographical affinity in brackets after the name of the species (DS: secondary freshwater, ER: estuarine resident, EU: marine euryhaline, ES: marine stenohaline). N: number of organisms; LP: Standard length; $S_{\mathrm{OC}}$ : Occurrence Index (R: rare, O: occasional, C: common, D: dominant)

\begin{tabular}{|c|c|c|c|c|c|c|}
\hline Taxón & $\mathbf{N}$ & LP & $\mathrm{cm})$ & & & $S_{\mathrm{c}}$ \\
\hline & & Mín. & Máx. & Mín. & Máx. & \\
\hline
\end{tabular}

\section{CLASE CHONDRICHTHYES}

ORDEN Torpediniformes

Familia NARCINIDAE

Narcine vermiculata Breder, 1928 [ES]

$1 \quad-\quad 23.7$

120 $\mathrm{R}$ 


\begin{tabular}{lllll}
\hline Taxón & N & LP $(\mathrm{cm})$ & Peso (g) & $S_{\mathrm{OC}}$ \\
\hline
\end{tabular}

ORDEN Rajiformes

Mín. Máx. Mín. Máx.

Familia RHINOBATIDAE

$\begin{array}{lllllllll}\text { Pseudobatos leucorhynchus (Günther,1867) [ES] } & 3 & 24.4 & 35.5 & 64 & 123 & \text { O }\end{array}$

Familia POTAMOTRYGONIDAE

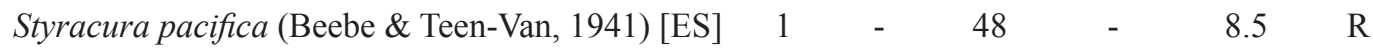

Familia GYMNURIDAE

Gymnura marmorata (Cooper, 1864) [ES]

$1 \quad-\quad 13.2 \quad-\quad 70 \quad \mathrm{R}$

CLASE ACTINOPTERYGII

ORDEN Albuliformes

Familia ALBULIDAE

Albula esuncula (Garman, 1899) [ES]

13

ORDEN Anguilliformes

Familia OPHICHTHIDAE

Bascanichthys bascanoides Osburn \& Nichol, $\quad 1 \quad-\quad 69.4 \quad-\quad 606$ R 1916 [ES]

Ethadophis byrnei Rosenblatt \& MCCosker, $1970 \quad 1 \quad-\quad \begin{array}{llllllll}76.5 & - & 49.70 & \text { R }\end{array}$ [ES]

Myrophis vafer Jordan \& Gilbert, 1883 [ES]

$\begin{array}{llllll}5 & 35.1 & 40.8 & 33.67 & 39.72 & \mathrm{O}\end{array}$

Myrichthys xysturus (Jordan \& Gilbert, 1882) [ES]

247.5

Ophichthus longipenis McCosker \& Rosenbla$\mathrm{tt}, 1998$ [ES]

Ophichthus zophochir Jordan \& Gilbert, 1882 [EU]

ORDEN Clupeiformes

Familia ENGRAULIDAE

Anchoa ischana Jordan \& Gilbert,1882 [EU]

$\begin{array}{llllll}19 & 5.9 & 9.8 & 1.24 & 5.01 & \mathrm{O}\end{array}$

Anchoa spinifer (Valenciennes, 1848) [EU]

6

Anchovia macrolepidota (Kner,1863) [ES]

\section{5}

Familia CLUPEIDAE

Lile gracilis Castro-Aguirre \& Vivero, 1990 [ER] $\quad \begin{array}{lllllll}13 & 6 & 9.8 & 1.53 & 5.69 & \text { O }\end{array}$

Opisthonema libertate (Günther,1867) [ES]

Opisthonema medirastre Berry \& Barret, 1963

$\begin{array}{llllll}6 & 15 & 20.8 & 63.33 & 79.85 & \mathrm{O}\end{array}$

[ES]

$\begin{array}{llllll}4 & 15.8 & 20.4 & 57.79 & 73.46 & \mathrm{R}\end{array}$

ORDEN Siluriformes

Familia ARIIDAE

Ariopsis guatemalensis (Günther, 1864) [EU] 


\begin{tabular}{|c|c|c|c|c|c|c|}
\hline \multirow[t]{2}{*}{ Taxón } & \multirow[t]{2}{*}{$\mathbf{N}$} & \multicolumn{2}{|c|}{$\mathbf{L P}(\mathbf{c m})$} & \multicolumn{2}{|c|}{ Peso (g) } & \multirow[t]{2}{*}{$\boldsymbol{S}_{\mathrm{OC}}$} \\
\hline & & Mín. & Máx. & Mín. & Máx. & \\
\hline Cathorops fuerthii (Steindachner, 1877) [EU] & 1 & - & 18.6 & - & 79.62 & $\mathrm{R}$ \\
\hline \multicolumn{7}{|l|}{ ORDEN Aulipiformes } \\
\hline \multicolumn{7}{|l|}{ Familia SYNODONTIDAE } \\
\hline Synodus scituliceps Jordan \& Gilbert, 1882 [ES] & 1 & - & 21.8 & - & 74.11 & $\mathrm{R}$ \\
\hline \multicolumn{7}{|l|}{ ORDEN Brachoidiformes } \\
\hline \multicolumn{7}{|l|}{ Familia BATRACHIDIDAE } \\
\hline $\begin{array}{l}\text { Batrachoides boulengeri Gilbert \& Starks, } 1904 \\
\text { [EU] }\end{array}$ & 1 & - & 10.5 & - & 13 & $\mathrm{R}$ \\
\hline
\end{tabular}

ORDEN Mugiliformes

Familia MUGILIDAE

Mugil curema Valenciennes, 1836 [EU]

$\begin{array}{llllll}11 & 4.7 & 20.9 & 0.66 & 64.59 & \mathrm{C}\end{array}$

ORDEN Atheriniformes

Familia ATHERINOPSIDAE

$\begin{array}{llllllll}\text { Atherinella guatemalensis (Günther, 1864) [ER] } & 5 & 5.8 & 8.3 & 0.5 & 1.02 & \mathrm{O}\end{array}$

ORDEN Beloniformes

Familia HEMIRAMPHIDAE

$\begin{array}{lllllll}\text { Hyporhamphus unifasciatus (Ranzani, 1841) [EU] } & 6 & 13.2 & 21.1 & 7.98 & 20.10 & \mathrm{R}\end{array}$

Familia BELONIDAE

Tylosurus fodiator Jordan \& Gilbert,1882 [ES] $\quad \begin{array}{lllllll} & 9 & 34 & 43.7 & 48 & 95.38 & \text { O }\end{array}$

ORDEN Cyprinodontiformes

Familia POECILIIDAE

Poecilia sphenops Valenciennes, 1846 [DS]

Poeciliopsis fasciata (Meek, 1904) [DS]

Poeciliopsis turrubarensis (Meek, 1912) [DS]

ORDEN Gasterosteiformes

Familia SYNGNATHIDAE

Hippocampus ingens Girard, 1858 [ES]

Pseudophallus starksii (Jordan \& Culver, 1895)

[ER]

$\begin{array}{cccccc}27 & 5.3 & 8.8 & 2 & 5.8 & \mathrm{R} \\ 1 & - & 5.1 & - & 3 & \mathrm{R} \\ 4 & 5.8 & 8.1 & 1 & 4 & \mathrm{R}\end{array}$

ORDEN Scorpaeniformes

Familia SCORPAENIDAE

Scorpaena mystes Jordan \& Stark, 1895 [ES]

$1 \quad-\quad 18.6 \quad-\quad 285 \quad \mathrm{R}$

Familia TRIGLIDAE

Prionotus horrens (Richardson, 1844) [ES]

ORDEN Perciformes 


\section{Taxón}

\begin{tabular}{ccccc} 
N & LP (cm) & \multicolumn{2}{c}{ Peso (g) } & $S_{\mathrm{OC}}$ \\
Mín. & Máx. & Mín. $\quad$ Máx.
\end{tabular}

Familia CENTROPOMIDAE

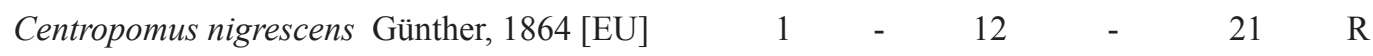

$\begin{array}{lllllll}\text { Centropomus robalito Jordan \& Gilbert, } 1882[\mathrm{EU}] & 25 & 5.9 & 20.3 & 2.08 & 69.05 & \mathrm{C}\end{array}$

Familia CARANGIDAE

$\begin{array}{lllllll}\text { Carangoides vinctus Jordan \& Gilbert,1882 [ES] } & 3 & 7.2 & 9.3 & 5.29 & 6.61 & \text { O }\end{array}$

Caranx caninus Günther, 1867 [EU]

Caranx sexfasciatus Quoy \& Gaimard, 1825 [ES]

Chloroscombrus orqueta Jordan \& Gilbert, 1883

[EU]

Oligoplites altus (Günther, 1868) [EU]

Oligoplites saurus (Bloch \& Schneider,1801) [EU]

Selene peruviana Steindachner, 1881 [ES]

$2 \quad 8.8 \quad 16.1$

9.70

$39.01 \quad \mathrm{R}$

$\begin{array}{lllllll}1 & - & 7.9 & - & 8 & \mathrm{R}\end{array}$

$\begin{array}{llllll}8 & 13.9 & 18.9 & 25.03 & 47.90 & \mathrm{O}\end{array}$

Familia LUTJANIDAE

Hoplopagrus guentherii Gill,1862 [ES]

Lutjanus argentiventris (Peters,1869) [ES]

Lutjanus guttatus (Steindachner, 1869) [ES]

Lutjanus novemfasciatus Gill, 1862 [EU]

Familia GERREIDAE

Diapterus brevirostris (Sauvage, 1879) [EU]

Eucinostomus currani Zahuranec, 1980 [EU]

Eucinostomus dowii (Gill, 1863) [EU]

Eucinostomus gracilis (Gill, 1862) [EU]

Eugerres lineatus ( Humboldt, 1821) [ES]

Gerres simillimus Regan, 1907 [EU]

Familia HAEMULIDAE

Pomadasys macracanthus(Günther, 1864) [EU]

Familia POLYNEMIDAE

Polydactylus approximans (Lay \& Bennett, 1839) [ES]

$\begin{array}{cccccc}25 & 4.9 & 24.1 & 0.86 & 89 & \mathrm{O} \\ 6 & 4.3 & 19.9 & 0.48 & 33.06 & \mathrm{C} \\ 5 & 6 & 17 & 2.14 & 38.67 & \mathrm{O}\end{array}$

$\begin{array}{cccccc}3 & 14.2 & 26.9 & 123 & 375 & \mathrm{O} \\ 3 & 6.7 & 17.9 & 5 & 75.25 & \mathrm{O} \\ 1 & - & 10.8 & - & 20.93 & \mathrm{R} \\ 1 & - & 14.8 & - & 73 & \mathrm{R}\end{array}$

Familia SCIAENIDAE

Micropogonias altipinnis (Günther, 1864) [EU]

Bairdiella armata (Gill, 1863) [ES]

$\begin{array}{ccc}27 & 11.3 & 23.2 \\ 1 & - & 10.3\end{array}$

$29.62 \quad 112.16 \quad \mathrm{C}$

Familia KYPHOSIDAE

Kyphosus vaigiensis (Quoy \& Gaimard, 1825)

[ES]

$\begin{array}{cccccc}25 & 7.5 & 14.7 & 8 & 32.93 & \mathrm{C} \\ 149 & 3.9 & 14.6 & 0.59 & 37.13 & \mathrm{D} \\ 58 & 5.5 & 12.9 & 2.12 & 20.84 & \mathrm{C} \\ 74 & 4.8 & 15.1 & 1.14 & 47.85 & \mathrm{D} \\ 1 & - & 8.1 & - & 13.01 & \mathrm{R} \\ 35 & 4.3 & 22.4 & 1 & 106 & \mathrm{O}\end{array}$

$\begin{array}{llllll}5 & 4.4 & 30.5 & 1.11 & 47.85 & \mathrm{O}\end{array}$

$1 \quad-\quad 15.3 \quad-\quad 70.04 \quad \mathrm{R}$

$-10.3 \quad . \quad 17.68 \mathrm{R}$

$\begin{array}{llllll}3 & 14.5 & 19.2 & 82 & 103.17 & \text { O }\end{array}$ 


\begin{tabular}{|c|c|c|c|c|c|c|}
\hline \multirow[t]{2}{*}{ Taxón } & \multirow[t]{2}{*}{$\mathbf{N}$} & \multicolumn{2}{|c|}{$\mathbf{L P}(\mathbf{c m})$} & \multicolumn{2}{|c|}{ Peso (g) } & \multirow[t]{2}{*}{$\boldsymbol{S}_{\mathrm{OC}}$} \\
\hline & & Mín. & Máx. & Mín. & Máx. & \\
\hline \multicolumn{7}{|l|}{ Familia CHAETODONTIDAE } \\
\hline Chaetodon humeralis Günther, 1860 [ES] & 2 & 6.5 & 8.3 & 8.68 & 12.33 & $\mathrm{R}$ \\
\hline \multicolumn{7}{|l|}{ Familia CICHLIDAE } \\
\hline Amphilophus trimaculatus(Günther, 1867) [DS] & 76 & 4 & 18.3 & 1 & 24.85 & $\mathrm{O}$ \\
\hline \multicolumn{7}{|l|}{ Familia ELEOTRIDAE } \\
\hline Dormitator latifrons (Richardson, 1844) [ER] & 13 & 5 & 15.8 & 2 & 54.75 & $\mathrm{O}$ \\
\hline $\begin{array}{l}\text { Erotelis armiger (Jordan \& Richardson, 1895) } \\
\text { [EU] }\end{array}$ & 7 & 8.2 & 21.4 & 2.93 & 11.53 & $\mathrm{O}$ \\
\hline Gobiomorus maculatus (Günther, 1859) [ER] & 1 & - & 10.4 & - & 2 & $\mathrm{R}$ \\
\hline \multicolumn{7}{|l|}{ Familia GOBIIDAE } \\
\hline Ctenogobius sagittula (Günther,1861) [EU] & 17 & 5.9 & 17.8 & 1.40 & 14.54 & $\mathrm{O}$ \\
\hline Gobionellus microdon Gilbert, 1892 [ER] & 55 & 5.2 & 18.3 & 1.05 & 24.45 & $\mathrm{D}$ \\
\hline \multicolumn{7}{|l|}{ Familia EPHIPPIDAE } \\
\hline Chaetodipterus zonatus (Girard, 1858) [ES] & 1 & - & 11.2 & - & 68.98 & $\mathrm{R}$ \\
\hline \multicolumn{7}{|l|}{ ORDEN Pleuronectiformes } \\
\hline \multicolumn{7}{|l|}{ Familia PARALICHTHYIDAE } \\
\hline $\begin{array}{l}\text { Citharichthys gilberti Jenkins \& Evermann, } 1889 \\
\text { [EU] }\end{array}$ & 7 & 10.7 & 22.6 & 15.57 & 104.57 & $\mathrm{O}$ \\
\hline Etropus crossotus Jordan \& Gilbert, 1882 [EU] & 13 & 6.6 & 16.3 & 2.69 & 42.11 & $\mathrm{O}$ \\
\hline \multicolumn{7}{|l|}{ Familia ACHIRIDAE } \\
\hline Achirus mazatlanus (Steindachner, 1869) [EU] & 51 & 3.6 & 11.9 & 0.47 & 22.62 & $\mathrm{D}$ \\
\hline Achirus scutum (Günther, 1862) [ES] & 11 & 4 & 10.7 & 0.75 & 14.25 & $\mathrm{O}$ \\
\hline \multicolumn{7}{|l|}{ Familia CYNOGLOSSIDAE } \\
\hline Symphurus elongatus (Günther, 1868) [ES] & 14 & 9.4 & 14.9 & 6.82 & 22.98 & $\mathrm{O}$ \\
\hline
\end{tabular}

ORDEN Tetraodontifomes

Familia BALISTIDAE

$\begin{array}{lllllllll}\text { Pseudobalistes naufragium (Jordan \& Starks, } & 1 & - & 12 & - & 107.35 & \mathrm{R}\end{array}$ 1895) [ES]

Familia TETRAODONTIDAE

Sphoeroides annulatus (Jenyns, 1842) [EU]

Familia DIODONTIDAE

Diodon holocanthus Linnaeus, 1758 [ES]

Diodon hystrix Linnaeus, 1758 [EU]

$\begin{array}{cccccc}13 & 3.9 & 18.1 & 0.92 & 106.52 & \mathrm{C} \\ & & & & & \\ 9 & 12.5 & 26.8 & 132.44 & 502 & \mathrm{O} \\ 1 & - & 24 & - & 503 & \mathrm{R}\end{array}$


indicadores de diversidad mostraron sus valores más altos durante el mes de noviembre $\left(K=0.93 ; \mathrm{H}^{\prime}=2.91\right)$, mientras que los más bajos se calcularon para enero $\left(\Lambda=0.87 ; \mathrm{H}^{\prime}=2.42\right.$; Fig. 4$)$.

En este estudio se reportan 15 nuevos registros de peces para la laguna Mar Muerto, de los cuales, cuatro se consideran nuevas adiciones a la ictiofauna continental de Chiapas: Narcine vermiculata Breder, 1928, Pseudobatos leucorhynchus (Günther,1867), Gymnura marmorata
(Cooper, 1864), Anchoa spinifer (Valenciennes, 1848), y seis no se habían considerado previamente como parte de la ictiofauna marina y estuarina estatal: Bascanichthys bascanoides Osburn \& Nichol, 1916; Ethadophis byrnei Rosenblatt \& McCosker, 1970; Myrophis vafer Jordan \& Gilbert, 1883; Ophichthus longipenis McCosker \& Rosenblatt,1998; Scorpaena mystes Jordan \& Stark, 1895; Prionotus horrens (Richardson, 1844) (Cuadro 3).
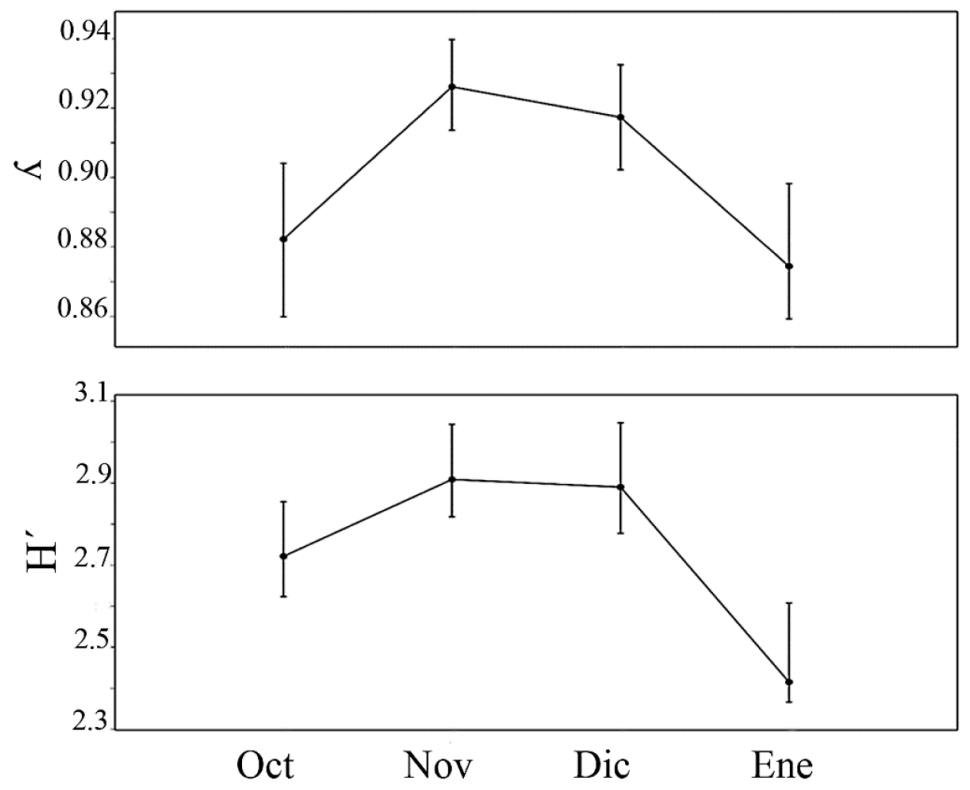

Fig. 4. Variación mensual de la diversidad: Índice inverso de Simpson $(\Lambda)$ e Índice de Shannon-Wiener $\left(\mathrm{H}^{\prime}\right)$

Fig. 4. Monthly variation of diversity: Inverse Simpson Index $(\Lambda)$ and ShannonWiener Index $\left(\mathrm{H}^{\prime}\right)$ 
Cuadro 3. Nuevos registros locales y regionales de especies de peces obtenidos de la ictiofauna de descarte de una pesquería con copo en la laguna costera Mar Muerto durante el periodo octubre 2017-enero 2018. *Catálogo. Museo de Zoología de la Universidad de Ciencias y Artes de Chiapas (Tuxtla Gutiérrez, Chiapas, México), Registro de Colección Científica: CHIS-PEC-210-03-09

Table 3. New local and regional records of fish species obtained from discarded ichthyofauna in cod-end net fishery in the Mar Muerto coastal lagoon during the October 2017-January 2018 period. *Catalogue. Museum of Zoology of the University of Sciences and Arts of Chiapas (Tuxtla Gutiérrez, Chiapas, Mexico), Scientific Collection Records: CHIS-PEC-210-03-09

\begin{tabular}{|c|c|c|c|c|c|}
\hline Especie & 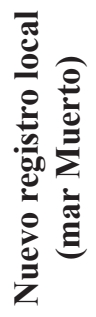 & 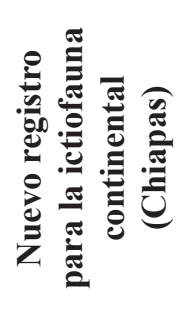 & 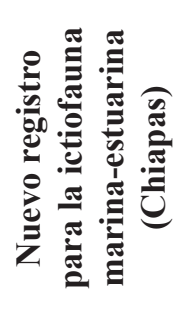 & 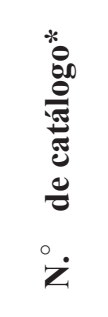 & 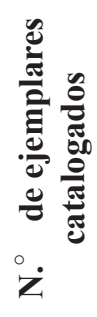 \\
\hline Narcine vermiculata & & $\checkmark$ & & 7284 & 1 \\
\hline Pseudobatos leucorhynchus & & $\checkmark$ & & 7339 & 3 \\
\hline Gymnura marmorata & & $\checkmark$ & & 7286 & 1 \\
\hline Bascanichthys bascanoides & & & $\checkmark$ & 7287 & 1 \\
\hline Ethadophis byrnei & & & $\checkmark$ & 7295 & 1 \\
\hline Myrophis vafer & & & $\checkmark$ & 7288 & 5 \\
\hline Ophichthus longipenis & & & $\checkmark$ & 7291 & 1 \\
\hline Anchoa spinifer & & $\checkmark$ & & 7280 & 6 \\
\hline Cathorops fuerthii & $\checkmark$ & & & 7289 & 1 \\
\hline Tylosurus fodiator & $\checkmark$ & & & 7290 & 4 \\
\hline Hippocampus ingens & $\checkmark$ & & & 7282 & 1 \\
\hline Scorpaena mystes & & & $\checkmark$ & 7292 & 1 \\
\hline Prionotus horrens & & & $\checkmark$ & 7283 & 1 \\
\hline Caranx sexfasciatus & $\checkmark$ & & & 7293 & 1 \\
\hline Diodon holocanthus & $\checkmark$ & & & 7338 & 1 \\
\hline
\end{tabular}




\section{DISCUSIÓN}

El presente análisis aportó información base sobre el descarte asociado a una pesquería ribereña de camarón no regulada. Con respecto a la proporción de descarte/camarón estimada durante el periodo de estudio, se encontró una proporción promedio de 2.6:1, con una mínima para el mes de diciembre $(1.25: 1)$ y una máxima durante octubre $(6.17: 1)$. Aunque estas correspondencias son menores a las reportadas para pesca de camarón por arrastre en áreas cercanas a la línea de la costa y la plataforma continental (Alverson et al. 1994; Chen et al. 2013; Al-Mamry et al. 2015), pueden considerarse altas para el caso específico de la pesca artesanal con artes fijas al interior de ambientes continentales. Por ejemplo, en la laguna costera de Chabihau, al norte de la península de Yucatán, reportaron una proporción promedio de descarte/camarón de 1:7, al utilizar una red de fondo fija (triángulo camaronero) (Leal et al. 2009). Los resultados de nuestro estudio coinciden con los obtenidos por López-Vila (2010) para pesca con copo en un sistema lagunar del sur de Chiapas, reportándose una proporción promedio de 2.8:1.

Aunque la mayoría de los estudios registraron variaciones temporales en la biomasa, tanto de la fauna de acompañamiento como del descarte (Maharaj \& Recksiek, 1991; Uzer et al. 2017), es necesario considerar, que para este trabajo, el muestreo se limitó a los cuatro meses que comprenden la pesca con copo en el Mar Muerto. Durante este periodo, las condiciones ambientales presentan relativa homogeneidad en la Boca de Tonalá (Tapia-García et al. 2011); sin embargo, el efecto de los escurrimientos estacionales al inicio de la temporada de pesca influye en los hábitos de reproducción y migración de muchas especies (Amezcua et al. 2006), lo que puede favorecer un marcado pico de biomasa y variación en la proporción durante el mes de octubre. Este patrón también ha sido reportado por López-Vila (2010) y Gómez-González (2010) en el sistema lagunar estuarino Chantuto-Pazacola, en la costa de Chiapas.

En este estudio se encontró que la ictiofauna integró a más de la mitad del descarte total, entre un $51 \%$ en diciembre y un $79.5 \%$ en octubre, lo cual es coincidente con lo reportado en otras pesquerías artesanales de camarón que utilizan copos, o variaciones de este, en lagunas costeras (Leal et al. 2009; López-Vila, 2010). Otros componentes de la fauna descartada en este estudio fueron, principalmente, jaibas (Callinectes spp.) y en menor proporción cefalópodos (probablemente del género Lolliguncula), sipuncúlidos, anélidos, bivalvos e incluso neonatos de tortuga golfina (Lepidochelys olivacea). En el caso de las jaibas, estas suelen ser un componente importante en las capturas 
incidentales asociadas a la pesca de camarón en México (De la Rosa-Meza, 2005; INAPESCA, 2012; WakidaKusunoki et al. 2013). En este trabajo se observaron jaibas de diferentes tamaños en abundante proporción durante los meses de noviembre y diciembre. Las jaibas del género Callinectes, aún las de tamaño pequeño, son depredadoras de camarones (Mascaró et al. 2003); y su abundancia, al igual que la de otros peces, podría estar relacionada con la disponibilidad de este recurso alimenticio. Por otro lado, la ausencia de una relación aparente entre la biomasa de camarón y la de ictiofauna de descarte puede deberse a la presencia de organismos adultos de tallas pequeñas, juveniles y subadultos $(9.9 \pm 9 \mathrm{~cm}$, longitud patrón) con limitado potencial depredador hacia el camarón, así como a la remoción de ejemplares con valor comercial (Milisenda et al. 2017; Öndes et al. 2017).

Algunos organismos que integran la ictiofauna de acompañamiento son consumidos o comercializados, aunque se considera que el porcentaje de su aprovechamiento es usualmente bajo (entre $8 \%$ y 18\%) (Amezcua et al. 2006). En este análisis se observó la selección de peces pertenecientes a especies como Albula esuncula, Mugil curema, Hoplopagrus guentherii y Micropogonias altippinis, cuyos ejemplares alcanzaban tallas mayores a $30 \mathrm{~cm}$, que por lo general, son utilizados para autoconsumo. La mayor parte de los peces óseos que componen el descarte en pesquerías artesanales de camarón, corresponden a individuos juveniles, reportándose, para un sistema costero del Pacífico mexicano, tallas mínimas y máximas de entre 2.9 y $17.8 \mathrm{~cm}$ (Estrella-Inzunza \& DíazGaxiola, 2017). Aunque, con respecto a los informes de tallas, es necesario considerar las características biológicas de cada especie y su densidad, ya que está demostrado que los ambientes costeros representan importantes áreas de crianza para peces juveniles o de primera madurez (Verdiell-Cubedo et al. 2013; Tournois et al. 2017).

La ictiofauna que integró el descarte fue desechada al interior de la laguna una vez concluida la selección del camarón y otros organismos aprovechables. Se observó que la mayoría de los peces se encontraban muertos en ese momento, probablemente como consecuencia del tiempo que pasan expuestos en la superficie o por el daño físico asociado a las maniobras de pesca (Davis, 2002; Davis \& Parker, 2004). Por lo tanto, este tipo de actividad pesquera puede afectar directamente al reclutamiento de especies, muchas de las cuales pertenecen a familias de interés comercial local (e.g. Carangidae, Mugillidae, Gerridae, Scianiadae) (Clay, 1996, Damalas et al. 2015; Morán-Silva et al. 2017). Sin embargo, la mayoría de las pesquerías de camarón en el mundo carecen de estudios sobre sus descartes, que 
permitan estimar con más precisión su impacto en las poblaciones (Kelleher, 2005). Por otro lado, el descarte podría beneficiar a algunas especies de crustáceos, peces y aves oportunistas o de hábitos carroñeros (Svane et al. 2008; Jodice et al. 2011). Por ejemplo, López-Vila (2010) observó que debido al horario nocturno de las maniobras de pesca con copo en el sur de Chiapas, el descarte beneficia a una especie de murciélago piscívoro (Noctilio leporinus). Desde el punto de vista ecosistémico, gran parte del descarte podría incorporarse al ciclo de reciclado de materia al ser transformado en detritus, mediante la intervención de organismos descomponedores (Queirolo et al. 1995; Heath et al. 2014). No obstante, es necesario investigar más a fondo sobre el impacto del descarte en la dinámica trófica en zonas estuarinas (Manickchand-Heileman et al. 2004).

Debido al limitado número de estudios sobre la ictiofauna de descarte o acompañamiento en la pesca de camarones en lagunas costeras del Pacífico mexicano, en general, se carece de valores referentes sobre riqueza, abundancia, biomasa y composición, así como de atributos asociados a las poblaciones como edades o proporciones sexuales. En este estudio, un total de 73 especies de peces fueron obtenidas como descarte, en comparación a 173 especies en el sistema lagunar Santa María La Reforma, Golfo de California (Amezcua et al. 2006); 71 especies en el sistema lagunar Chantuto-Panzacola, Chiapas (López-Vila, 2010) y 14 especies en el sistema lagunar Topolobampo, Sinaloa (Estrella-Inzunza y Díaz-Gaxiola, 2017). Mientras que en el primer y último estudio referido, estas diferencias observadas pueden deberse a la variación en el grado de selectividad de las artes de pesca, en el segundo se aprecia una marcada similitud en el número de especies registradas. A pesar de encontrarse una riqueza relativamente alta en el descarte, es usual que se encuentran pocas especies y familias como dominantes. López-Vila (2010) encontró en el sistema Chantuto-Panzacola (localizado aproximadamente $150 \mathrm{~km}$ al sur de la boca de Mar Muerto) que organismos de las especies Cathorops liropus (Ariidae) y Dormitator latifrons (Eleotridae) integraban más del $26 \%$ de la abundancia numérica del descarte por pesca con copo. En contraste, en este estudio se encontró a tres especies del género $E u$ cinostomus (Gerreidae) que representa a más del $30 \%$ del total de individuos muestreados. Estas diferencias taxonómicas pueden deberse a los patrones de reclutamiento, reproducción y migración de las especies abundantes (Amezcua et al. 2006), asociados a las condiciones ambientales intrínsecas y a las variaciones locales de la pesca con copo.

En el sistema ChantutoPanzacola, esta actividad pesquera se realiza, principalmente, asociada a la temporada lluviosa, cuando se 
obtienen los mayores volúmenes de camarón (López-Vila, 2010); podrían las condiciones oligohalinas del sistema favorecer la presencia de especies ícticas estuarinas y dulceacuícolas secundarias. En el caso del sistema Mar Muerto, un menor aporte de agua dulce y la marcada influencia marina (debido a la cercanía de los sitios de muestreo con la bocabarra) pueden determinar una mayor dominancia de especies estenohalinas y eurihalinas (RomeroBerny et al. 2018). Lo anterior quedó claramente evidenciado en el presente estudio, en donde el componente marino representó el $86.3 \%$ de la ictiofauna descartada. Así mismo, los mayores valores de diversidad se presentaron durante el periodo de transición hidrológica lluvias-secas (noviembrediciembre) que afecta la salinidad e incrementa el número de especies raras de afinidad marina dentro del sistema.

Con respecto a la categoría de ocurrencia, los resultados muestran que más del $80 \%$ de las especies presentaron patrones de dominancia como raras y ocasionales, lo cual es una característica común en comunidades de peces en lagunas costeras (Acuña et al. 2017). No obstante, es necesario considerar las restricciones en el tamaño de la muestra, por lo que es recomendable ampliar el estudio hacia series de tiempo, a fin de tener una estimación más precisa de estos patrones.

En este estudio se registró al $66.3 \%$ de las especies previamente re- portadas para el sistema Mar Muerto. A pesar de utilizarse una sola arte de pesca, el número de especies de peces encontradas y la adición de 15 nuevos registros representan un resultado notable en la generación de nueva información sobre composición en el nivel local y regional. Los inventarios de ictiofauna realizados en lagunas costeras del norte del Golfo de Tehuantepec han sido escasos y dispersos (Chávez, 1979; Castro-Aguirre, 1982; Tapia-García et al. 1998; Tapia-García y Mendoza-Rodríguez, 2005; Romero-Berny et al. 2018). El sistema Mar Muerto destaca en esta área del Golfo por presentar la mayor riqueza íctica reportada, cuyo número se incrementa a 108 especies al considerar los registros de este estudio. Es probable que la elevada riqueza del sistema se encuentre relacionada con la amplitud de la boca y morfología de la laguna, factores que favorecen una conectividad permanente con el ambiente oceánico (Mendoza et al. 2009). Es interesante mencionar que cuatro de los nuevos registros (27\%) corresponden a la familia Ophichthidae, la cual se encontraba poco representada en los ambientes costeros continentales de Chiapas (Velázquez-Velázquez et al. 2016). También, es importante destacar el papel de los estudios sobre fauna acompañante de la pesca en la ampliación del conocimiento de la diversidad íctica (López-Martínez et al. 2010). 


\section{CONCLUSIONES}

Este trabajo representa una de las primeras aproximaciones a la caracterización del descarte en una pesquería artesanal de camarón, escasamente evaluada en sistemas costeros continentales del Pacífico sur mexicano. En este trabajo se demostró que la pesca por copo afecta potencialmente por lo menos a 73 especies de peces que pueden tratarse, de acuerdo a la talla promedio (9.9 $\pm 9 \mathrm{~cm}$, longitud patrón) $\mathrm{y}$ especie, de individuos juveniles y sub-adultos; en una proporción general de ictiofauna/camarón de 1.89:1, considerada alta en comparación a otros estudios. Aunque en términos de estimación de proporciones, composición y riqueza ictiofaunística es una importante línea base, este trabajo aún es insuficiente para comprender integralmente la dinámica ecológica de las especies involucradas. Es recomendable enfocar los esfuerzos de investigación en determinar las variables ambientales (p. ej. salinidad, oxígeno disuelto, velocidad de corriente, temperatura del agua) y biológicas (p. ej. diversidad, composición taxonómica y funcional, madurez gonádica, migraciones, dietas) que mejor definen la estructura de la fauna de acompañamiento y descarte. Así mismo, es necesario contar con evaluaciones en series interanuales para tener un conocimiento más preciso sobre las especies aprovechadas, desechadas y el manejo aplicado al descarte. Al tratarse de una pesquería ilegal, la obtención de esta información es difícil. Por lo tanto, es necesario realizar un acercamiento con los grupos de pescadores usuarios de copo, a fin de establecer mecanismos de regulación orientados a la reducción y un manejo adecuado de las capturas secundarias.

\section{BIBLIOGRAFÍA}

Acuña, P. A., Gurdek, R., Muñoz, N., Gutiérrez, J. M., Spósito, M., Correa, P. \& Caride, A. (2017). Seasonal composition, abundance and biomass of the subestuarine fish assemblage in Solís Chico (Río de la Plata estuary, Uruguay). Braz. J. Biol., 77(3), 622-631.

Allen, R. G. \& Robertson, D. R. (1998). Peces del Pacífico oriental tropical. México, D. F.; CONABIO/Agrupación Sierra Madre/CEMEX.

Al-Mamry, J. M., Chesalin, M. V., Al-Mamary, D. S. \& Al-Senaidi, R. H. (2015). The bycatch composition of shrimp trawl fishery in the Oman coastal waters, the Arabian Sea. Acta Ichthyol. Piscat., 45(3), 273-283. https://doi. org/10.3750/AIP2015.45.3.06

Alverson, D. L., Freeberg, M. H., Murawski, S. A. \& Pope, J. G. (1994). A Global Assessment of Fisheries Bycatch and Discards. FAO. Doc. Tec. Pesca 339. Roma, Italia: FAO.

Amezcua, F., Madrid-Vera, J. \& Aguirre-Villaseñor, H. (2006). Efecto de la pesca artesanal de camarón sobre la ictiofauna en el sistema lagunar de Santa María la Reforma, suroeste del Golfo de California. Ciencias Marinas, 32, 
97-109. https://doi.org/10.7773/cm. v32i12.1025

Amezcua, F., Madrid-Vera, J. \& Aguirre-Villaseñor, H. (2009). Incidental capture of juvenile fish from an artisanal fishery in a coastal lagoon in the Gulf of California. N. Am. J. Fish. Manage., 29, 245-255. https://doi.org/10.1577/ M06-022.1

Bojórquez, L. L. F. (1999). Shrimp bycatch utilization in Mexico. In I. Clucas, \& F. Teutscher, (Eds.), Bycatch utilization in tropical fisheries (pp. 253-262). Roma, Italia: FAO.

Burgos-León, B., Pérez-Castañeda, R. \& Defeo, O. (2009). Discards from the artisanal shrimp fishery in a tropical coastal lagoon of Mexico: spatio-temporal patterns and fishing gear effects. Fisheries Manag. Ecol., 16, 130-138. https://doi. org/10.1111/j.1365-2400.2009.00653.x

Bussing, W. A. \& López, M. I. (2011). Peces demersales y pelágicos costeros del Pacífico de Centroamérica meridional. San José, Costa Rica: Universidad de Costa Rica.

Castro-Aguirre, J. L. (1982). Los peces de las lagunas Oriental y Occidental, Oaxaca, México y sus relaciones con temperatura y salinidad. II. Análisis multifactorial. An. Esc. Nac. Cienc. Biol., 26(14), 85-100.

Castro-Aguirre, J. L., Espinosa-Pérez, H. \& Schmitter-Soto, J. J. (1999). Ictiofauna estuarino-lagunar y vicaria de México. México, D. F.: Noriega-Limusa.

Clay, J. (1996). Market potentials for redressing the environmental impact of wild captured and pond produced shrimp. Washington, D. C., EE. UU.: WWF.

Contreras, E. F. (2002). Importancia de la pesca ribereña en México. ContactoS, 46, 5-14.

Chávez, E. A. (1979). Análisis de la comunidad de una laguna costera en la costa sur occidental de México. An. Cent. Cienc. Mar Limnol., 6, 15-44.

Chen, W., Almatar, S., Alsaffar, E. \& Yousef, A. R. (2013). Retained and discarded bycatch from Kuwait's shrimp fishery. Aquat. Sci. Technol., 1(1), 86-100.

Damalas, D., Maravelias, C. D., Osio, G. C., Maynou, F., Sbrana, M., Sartor, P. \& Casey, J. (2015). Historical discarding in Mediterranean fisheries: a fisher's perception. ICES J. Mar. Sci. 72(9), 2600-2608.

Davis, M. W. (2002). Key principles for understanding fish bycatch discard mortality. Can. J. Fish. Aquat. Sci., 59, 1834-1843.

Davis, M. W. \& Parker, S. J. (2004). Fish size and exposure to air: Potential effects on behavioral impairment and mortality rates in discarded sablefish. N. Am. J. Fish. Manage., 24(2), 518-524.

Day, J. W., Hall, C. A. S., Kemp, W. M. \& Yañez-Arancibia, A. (1989). Estuarine Ecology. New York, EE. UU.: Wiley.

De la Rosa-Meza, K. (2005). Fauna de acompañamiento del camarón en Bahía Magdalena, B. C. S. México. Tesis de maestría no publicada. Centro Interdisciplinario de Ciencias Marinas-Instituto Politécnico Nacional, La Paz.

Dos Santos, M. N., Saldanha, H. J. \& García, A. (2002). Observations on by-catch from a tuna trap fishery off the Algarve (Southern Portugal). ICCAT, 54(5), 1726-1732.

Eayrs, S. (2007). Guía para reducir la captura de fauna incidental (bycatch) en las pesquerías por arrastre de camarón tropical. Roma, Italia: FAO.

Eschmeyer, W. N., Fricke, R. \& van der Laan, R. (2017). Catalog of Fishes: Genera, Species, References. California: California Academy of Sciences. Recuperado el 23 de febrero 2018, disponible en http://researcharchive.calacademy. 
org/research/Ichthyology/catalog/fishcatmai.asp.

Estrella-Inzunza, P. \& Díaz-Gaxiola, J. M. (2017). Análisis de los peces de acompañamiento del camarón (PAC) capturados con churupa en el sistema lagunar de Topolobampo, Sinaloa, México. Ra Ximhai, 13(3), 319-338.

FAO. (1996). Technical consultation on reduction of wastage in fisheries. Tokio, 28 de octubre-1 noviembre 1996, FAO Fisheries Report No. 547. Roma, Italia: FAO.

Fischer, W., Krupp, F., Schneider, W., Sommer, C., Carpenter, K. E. \& Niem, V. H. (1995). Guía FAO para la identificación de especies para los fines de la pesca, vols. II-III. Roma, Italia: FAO.

García, E. (2004). Modificaciones al sistema de clasificación climática de Köppen. México, D. F.: Instituto de Geografía-UNAM.

García, S. \& Le Reste, L. (1986). Ciclos vitales, dinámica, explotación y ordenación de las poblaciones de camarones peneidos costeros. FAO. Doc. Tec. Pesca 203. Roma, Italia: FAO.

Gillett, R. (2008). Global study of shrimp fisheries. Rome, Italy: FAO.

Giménez-Hurtado, E., Caridad-Pérez Marrero, A., Delgado-Miranda, G., Alonso-Domínguez, H. \& Villafuerte-Delgado, V. (2016). Comportamiento de la fauna acompañante en la pesca de camarón rosado (Farfantepenaeus notialis) en la plataforma suroriental de Cuba. Rev. Electrón. Vet., 17(11), 1-22.

Gómez-González, A. E. (2010). Comunidad de peces del sistema Chantuto-Panzacola, Reserva de la Biósfera La Encrucijada. Tesis de maestría no publicada. El Colegio de la Frontera Sur, San Cristóbal de las Casas.

González-Acosta, A. F., Rodiles-Hernández, R. \& González-Díaz, A. A. (2017).
Checklist of the marine and estuarine fishes of Chiapas, Mexico. Mar. Biodivers. http://dx.doi.org/10.1007/ s12526-016-0630-y

González-Zuarth, C. \& Álvarez, D. (2014). La conducta de los peces como bioindicadores de la presencia de estresores ambientales. En C. A. González-Zuarth, A. Vallarino, J. C. Pérez-Jiménez \& A. M. Low-Pfeng (Eds.), Bioindicadores: guardianes de nuestro futuro ambiental (pp. 397-420). México, D. F.: Ecosur/ INECC.

Hall, M. A. (1996). On bycatches. Rev. Fish. Biol. Fisher., 6, 319-352. https://doi. org/10.1007/BF00122585

Hall, M. A., Alverson, D. L. \& Metuzals, K. I. (2000). By-catch: problems and solutions. Mar. Pollut. Bull., 41, 204219. https://doi.org/10.1016/S0025326X(00)00111-9

Hammer, Ø., Harper, D. A. T. \& Ryan, P. D. (2001). PAST: Paleontological Statistics software package for education and data analysis. Palaeontol. Electron., 4(1), 9 .

Heath, M. R., Cook. R. M., Cameron, A. I., Morris, D. J. \& Speirs, D. C. (2014). Cascading ecological effects of eliminating fishery discards. Nat. Commun., 5, 1-8. https://doi.org/10.1038/ncomms4893

Herrera-Valdivia, E., López-Martínez, J., Castillo-Vargasmachuca, S. \& García-Juárez, A. R. (2016). Diversidad taxonómica y funcional en la comunidad de peces de la pesca de arrastre de camarón en el norte del Golfo de California, México. Rev. Biol. Trop., 64, 587-602. https://doi.org/10.15517/rbt. v63i3. 15852

Huidobro, C. L. (2000). Peces. En G. De la Lanza-Espino, S., Hernández-Pulido, \& J. L. Carbajal-Pérez (Eds.), Organismos indicadores de la calidad del agua 
y la contaminación (pp. 195-263). México, D. F.: Plaza y Valdéz/UNAM.

Hutchings, J. A. (2000). Collapse and recovery of marine fishes. Nature, 406, 882-885. https://doi.org/10.1038/35022565

INAPESCA. (2012). Plan de manejo de la pesquería de camarón del Pacífico mexicano. México: SAGARPA-CONAPESCA/Fundación Produce.

Jodice, P. G. R., Wickliffe, L. C. \& Sachs, E. B. (2011). Seabird use of discards from a nearshore shrimp fishery in the South Atlantic Bight, USA. Mar. Biol., 158, 2289. http://dx.doi.org/10.1007/ s00227-011-1733-4

Kelleher, K. (2005). Discards in the world's marine fisheries - an update. Rome, Italy: FAO.

Krebs, C. J. (2009). Ecology: the experimental analysis of distribution and abundance. San Francisco, EE. UU.: Pearson Benjamin Cummings.

Leal, S. A., Cabrera, M. A. \& Salas, S. (2009). Caracterización de la fauna incidental en la pesquería artesanal de camarón en la laguna de Chabihau, Yucatán, México. En Proceedings of the $61^{\text {st }}$ Gulf and Caribbean Fisheries Institute (pp. 163172). Gosier, Guadeolupe, French West Indies: GFCI.

López-Martínez, J., Herrera-Valdivia, E., Rodríguez-Romero, J. \& Hernández-Vásquez, S. (2010). Peces de la fauna de acompañamiento en la pesca industrial de camarón en el Golfo de California, México. Rev. Biol. Trop., 58(3), 925942.

López-Martínez, J. \& Morales-Bojórquez, E. (2012). Efectos de la pesca de arrastre en el Golfo de California. México, D. F.: Centro de Investigaciones Biológicas del Noroeste, S. C. y Fundación Produce Sonora.

López-Vila, J. M. (2010). El descarte en la pesca del camarón en un sistema lagu- nar de la Reserva de la Biósfera "La Encrucijada”, Chiapas, México. Tesis profesional no publicada. Universidad de Ciencias y Artes de Chiapas, Tuxtla Gutiérrez.

Maharaj, V. \& Recksiek, C. (1991). The bycatch from the artisanal shrimp trawl fishery, Gulf of Paria, Trinidad. Mar. Fish. Rev., 53(2), 9-15.

Manickchand-Heileman, S., Mendoza-Hill, J., Kong, A. L. \& Arocha, F. (2004). A trophic model for exploring possible ecosystem impacts of fishing in the Gulf of Paria, between Venezuela and Trinidad. Ecol. Model., 172, 307322. https://doi.org/10.1016/j.ecolmodel.2003.09.013

Mascaró, M., Hidalgo, L. H., Chiappa-Carrara, X. \& Simōes, N. (2003). Size-selecting foraging behaviour of blue crabs Callinectes sapidus (Rathbun), when feeding on mobile prey: Active and passive components of predation. Mar. Freshw. Behav. Phy., 36(3), 143-159. https://doi.org/10.1080/102362403100 01603224

Medina-Reyna, E. E. (1999). La retención de las artes de pesca de camarón como indicador de la estrategia pesquera en la laguna Mar Muerto de Oaxaca. Ciencia y Mar, 7, 13-22.

Mendoza, E., Castillo-Rivera, M., Zárate-Hernández, R. \& Ortiz-Burgos, S. (2009). Seasonal variations in the diversity, abundance, and composition of species in an estuarine fish community in the Tropical Eastern Pacific, Mexico. Ichthyol. Res., 56, 330-339. https://doi. org/10.1007/s10228-009-0102-5

Milisenda, G., Vitale, S., Massi, D., Enea, M., Gancitano, V., Giusto, G. B., Badalucco, C., Gristina, M., Garofalo, G. \& Fiorentino, F. (2017). Discard composition associated with the deep water rose shrimp fisheries (Parapenaeus longirostris, 
Lucas 1846) in the south-central Mediterranean Sea. Mediterr. Mar. Sci., 18, 53-63. https://doi.org/10.12681/ mms. 1787

Miller, R. R. (1966). Geographical distribution of Central American freshwater fishes. Copeia, 4, 773-802. https://doi. org/10.2307/1441406

Miller, R. R., Minckley, W. L. \& Norris, S. M. (2009). Peces dulceacuícolas de México. México, D. F.: CONABIO/ SIMAC /ECOSUR/Desert Fishes Council.

Morán-Silva, A., Chávez-López, R., Jiménez-Baldillo, M. de L., Cházaro-Olvera, S., Galindo-Cortes, G. \& Meiners-Mandujano, C. G. (2017). Análisis de la comunidad de peces de descarte en la pesca de arrastre de camarón (temporada de lluvias 2013) en la zona centro-sur del litoral veracruzano, México. Rev. Biol. Mar. Oceanog., 52, 551-566. https://doi.org/10.4067/ S0718-19572017000300012

Myers, G. S. (1949). Salt-tolerance of fresh-water fish groups in relation to zoogeographical problems. Bijdr. Dier$k d$., 28, 315-322.

Nelson, J. S. (2006). Fishes of the World. New York, EE. UU.: Wiley.

Öndes, F., Kaiser, M. J. \& Murray, L. G. (2017). Fish and invertebrate by-catch in the crab pot fishery in the Isle of Man, Irish Sea. J. Mar. Biol. Assoc. UK., 1-13. http://dx.doi.org/ 10.1017/ S0025315417001643

Pérez-Castañeda, R. \& Defeo, O. (2001). Population variability of four sympatric penaeid shrimps (Farfantepenaeus spp.) in a coastal lagoon of Mexico. Estuar. Coast. Shelf Sci., 52, 631-641. https://doi.org/10.1006/ecss.2001.0771

Poot-Salazar, A., Pérez-Casteñeda, R., Vega-Cendejas, M. E. \& Defeo, O. (2009). Assessing patterns of ichthyofauna discarded by an artisanal shrimp fishery through selectivity experiments in a coastal lagoon. Fish. Res., 97, 155-162. https://doi.org/10.1016/j.fishres.2009.02.001

Queirolo, L. E., Fritz, L. W., Livingston, P. A., Loefflad, M. R., Colpo, D. A. \& deReynier, Y. L. (1995). Bycatch, utilization, and discards in the commercial groundfish fisheries of the Gulf of Alaska, Eastern Bering Sea, and Aleutian Islands. Seattle, EE. UU.:Dep. Commer., NOAA Technical Memorandum NMFS.

Ramírez-Herrejón, J. P., Mercado-Silva, M., Medina-Nava, M. \& Domínguez-Domínguez, O. (2012). Validación de dos índices biológicos de integridad (IBI) en la subcuenca del río Angulo en el centro de México. Rev. Biol. Trop., 60(4), 1669-1685. https://doi. org/10.15517/rbt.v60i4.2160

Ramos-Cruz, S. (2011). Selectividad y estimación de un tamaño mínimo de malla para las atarrayas camaroneras en la laguna Mar Muerto, Oaxaca-Chiapas, México, con implicaciones para el manejo de la pesquería. Ciencia Pesquera, 19(1), 47-57.

Rodríguez-Romero, J., Hernández-Vásquez, S. \& López-Martínez, J. (2009). Desarrollo potencial de peces desaprovechados. Ciencia y Desarrollo, 35, 45-51.

Romero-Berny, E. I., Velázquez-Velázquez, E., Anzueto-Calvo, M. J., Urbina-Trejo, E. \& Schmitter-Soto, J. J. (2018). The fish fauna of three lagoon-estuarine systems in the northeastern Gulf of Tehuantepec, Mexican south Pacific. Rev. Mex. Biodivers., 89, 84-98. https://doi.org/10.22201/ ib.20078706e.2018.1.1927

Schultz, D. M., Bracken, W. E., Bosart, L. F., Hakim, G. J., Bedrick, M. A., Dickinson, M. J, \& Tyle, K. R. (1997). The 1993 Superstorm Cold Surge: Frontal 
structure, gap flow, and tropical impact. Mon. Weather Rev., 125, 5-39. https://doi.org/10.1175/1520-0493(199 7) $125<0005$ :TSCSFS $>2.0 . \mathrm{CO} ; 2$

Soykan, C. U., Moore, J. E., Zydelis, R., Crowder, L. B., Safina, C. \& Lewison, R. L. (2008). Why study by-catch? An introduction to the theme section on fisheries by-catch. Endanger. Species Res., 5, 91-102. https://doi.org/10.3354/ esr00175

Stata Corp. (2009). STATA 11 Base Reference Manual. Texas, EE. UU.: StataCorp LLC

Svane, I., Roberts, S. \& Saunders, T. (2008). Fate and consumption of discarded bycatch in the Spencer Gulf, prawn fishery, SouthAustralia. Fish. Res., 90, 158-169. https://doi.org/10.1016/j.fishres.2007.10.008

Tapia-García, M. \& M. García-Abad. (1998). Los peces acompañantes del camarón y su potencial como recurso en las costas de Oaxaca y Chiapas. En M. Tapia-García (Ed.), El Golfo de Tehuantepec: el ecosistema y sus recursos (pp. 179-196). México: UAM-I.

Tapia-García, M. \& Mendoza-Rodríguez, R. (2005). Composición y abundancia de la ictiofauna en las lagunas Superior e Inferior, Oaxaca. Actual. Biol., 82, 5765.

Tapia-García, M., Suárez-Nuñez, C., Cerdenares-Ladrón de Guevara, G., Macuitl-Montes, M.C. \& García-Abad, M.C. (1998). Composición y distribución de la ictiofauna en la Laguna del Mar Muerto, Pacífico mexicano. Rev. Biol. Trop., 46(2), 277-284.

Tapia-García, M., García-Abad, M. C., Carranza-Edwards, A. \& Vásquez-Gutiérrez, F. (2007). Environmental characterization of the continental shelf of the Gulf of Tehuantepec, Mexico. Geofis. Int., 46(4), 249-260.
Tapia-García, M., García-Abad, M. C., Penagos-García, F. E., Moreno-Ruíz, J. L., Juárez-Hernández, L. G., Ramírez-Gutiérrez, J. M. \& Herrera-Olayo, D. (2011). Subsistemas hidrológicos de la laguna Mar Muerto, Oaxaca-Chiapas, México. Lacandonia, 5(1), 97-112.

Tournois, J., Damaude, A. M., Ferraton, F., Aliaume, C., Mercier, L. \& McKenzie, D. J. (2017). Lagoon nurseries make major contribution to adult populations of a highly prized coastal fish. Limnol. Oceanogr. 62(3), 1219-1233. https:// doi.org/10.1002/lno.10496

Tsagarakis, K., Carbonell, A., Brčić, J., Bellido, J. M., Carbonara, P., Casciaro, L., Edridge, A., García, T., González, M., Šifner, S. K., Machias, A., Notti, E., Papantoniou, G., Sala, A., Škeljo, F., Vitale, S. \& Vassilopoulou, V. (2017). Old Info for a New Fisheries Policy: Discard Ratios and Lenghts at Discarding in EU Mediterranean Bottom Trawl Fisheries. Front. Mar. Sci., 4. 1-13. http:// dx.doi.org/10.3389/fmars.2017.00099

Uzer, U., Yildiz, T. \& Karakulak, F. S. (2017). Catch composition and discard of the boat seine in Istambul Strait (Turkey). Turk. J. Zool., 41, 702-713. https://doi. org/10.3906/zoo-1610-10

Velázquez-Velázquez, E., López-Vila, J. M., Gómez-González, A. E., Romero-Berny, E. I., Liévano-Trujillo, J. L. \& Matamoros, W. A. (2016). Checklist of the continental fishes of the state of Chiapas, Mexico, and their distribution. ZooKeys, 632, 99-120. https://doi. org/10.3897/zookeys.632.9747

Verdiell-Cubedo, D., Oliva-Paterna, F., Ruiz-Navarro, A. \& Torralva, M. (2013). Assesing the nursery role for marine fish species in a hypersaline coastal lagoon (Mar Menor, Mediterranean Sea). Mar. Biol. Res. 8, 739-748. https://doi.org/10.1080/17451000.201 3.765580 
Wakida-Kusunoki, A. T., Becerra-De la Rosa, I., González-Cruz, A. \& Amador-Del Ángel, L. E. (2013). Distribución y abundancia de la fauna acompañante del camarón en la costa de Tamaulipas, México (veda del 2005). Universidady Ciencia, 29(1), 75-86.
Zeller, D., Cashion, T., Palomares, M. \& Pauly, D. (2017). Global marine fisheries discards: a synthesis of reconstructed data. Fish Fish., 19, 30-39. https:// doi.org/10.1111/faf.12233 